\title{
High-performance phosphorene electromechanical actuators
}

\author{
Bozhao Wu $\mathbb{D}^{1}$, Hui-Xiong Deng ${ }^{2}$, Xiangzheng Jia (D) ${ }^{1}$, Langquan Shui $\mathbb{D}^{1}$, Enlai Gao ${ }^{1 凶}$ and Ze Liu ${ }^{1 凶}$
}

Phosphorene, a two-dimensional material that can be exfoliated from black phosphorus, exhibits remarkable mechanical, thermal, electronic, and optical properties. In this work, we demonstrate that the unique structure of pristine phosphorene endows this material with exceptional quantum-mechanical performance by using first-principles calculations. Upon charge injection, the maximum actuation stress is $7.0 \mathrm{GPa}$, corresponding to the maximum actuation strain as high as $36.6 \%$ that is over seven times larger than that of graphene (4.7\%) and comparable with natural muscle (20-40\%). Meanwhile, the maximum volumetric work density of phosphorene $\left(207.7 \mathrm{~J} / \mathrm{cm}^{3}\right)$ is about three orders of magnitude larger than natural muscle $\left(0.008-0.04 \mathrm{~J} / \mathrm{cm}^{3}\right)$ and approximately six times larger than graphene $\left(35.3 \mathrm{~J} / \mathrm{cm}^{3}\right)$. The underlying mechanism of this exceptional electromechanical performance in phosphorene is well revealed from the analysis of atomic structure and electronic structure. Finally, the influence of charge on the mechanical behaviors of phosphorene is examined by mechanical tests, indicating the sufficient structural integrity of phosphorene under the combined electromechanical loading. These findings shed light on phosphorene for promising applications in developing nanoelectromechanical actuators.

npj Computational Materials (2020)6:27; https://doi.org/10.1038/s41524-020-0297-6

\section{INTRODUCTION}

Developing artificial muscles that can mimic the behaviors of mammalian skeletal muscle has attracted much attention but remains a long-term challenge ${ }^{1-5}$. Natural muscles boast reversible mechanical responses in a large strain range under various complex loads ${ }^{6-9}$. To mimic natural muscles, the selected materials for artificial muscles must exhibit significant strokes, stress as well as volumetric work densities at quite fast responses ${ }^{10}$. To date, the extensively studied actuation materials include shape memory alloys, electroactive ceramics, and polymers ${ }^{11}$. Shape memory alloys have high work densities, but unpredictable deformation and slow responses ${ }^{1}$. Electroactive ceramics possess fast responses but their strokes are $<1 \%{ }^{12}$. Polymers, e.g., nylon, can generate large stroke under heat stimulation, while their performance is limited by the low heat transfer efficiency between actuation and relaxation $^{4,13}$.

With the increasing demands for nanoactuator devices and the discovery of two-dimensional materials $(2 \mathrm{DMs})^{14-18}, 2 \mathrm{DM}$ based actuators have attracted considerable interests. Remarkable achievements made by Liu and his coworkers ${ }^{19-21}$ demonstrate that graphene and graphene oxide (GO) exhibit extraordinary electromechanical performance. For example, as GO changes from a metastable clamped configuration to a more stable unzipped configuration, it generates a huge reversible stroke of $28 \%{ }^{20}$. Recently phosphorene arises after successful exfoliation from its bulk form (black phosphorus) ${ }^{22}$, and a lot of efforts have been made to investigate its structures, properties as well as applications. For example, phosphorene possesses remarkable in-plane mechanical anisotropy ${ }^{23-25}$, which is very different from the in-plane elastic isotropy of graphene ${ }^{26}$. Theoretical calculations demonstrated that the strains to failure (tensile stiffnesses) of phosphorene along the armchair and zigzag directions are as high as $30 \%(23 \mathrm{~N} / \mathrm{m})$ and $27 \%(92.3 \mathrm{~N} / \mathrm{m})$, respectively ${ }^{23,24}$. The high strain to failure but small in-plane stiffness as well as remarkable mechanical anisotropy makes phosphorene highly promising in developing high-performance actuators. However, the electromechanical performance of phosphorene has not been explored.

In this work, we investigate the electromechanical performance of pristine phosphorene by using first-principles calculations. The results demonstrate that the charging-induced maximum actuation strain of phosphorene is ultrahigh (36.6\%), which is much larger than that of graphene $(4.7 \%)$ and silicene $(2.5 \%)$. Meanwhile, the volumetric work density is theoretically calculated as $207.7 \mathrm{~J} / \mathrm{cm}^{3}$, much larger than the well investigated graphene $\left(54.1 \mathrm{~J} / \mathrm{cm}^{3}\right)$ and GO $\left(144.1 \mathrm{~J} / \mathrm{cm}^{3}\right)$. The underlying mechanism of this exceptional electromechanical performance in phosphorene is well revealed from its electronic and atomic structure analysis. Finally, the good structural integrity of phosphorene under the combined electromechanical loading is examined.

\section{RESULTS}

Electromechanical strain response of phosphorene

The models used in the calculations of electromechanical responses are the unit cells of these 2DMs (Fig. 1). Before charge injection, the fully structural optimizations were performed, resulting in optimized lattice constants $a_{1}=4.572 \AA, a_{2}$ $=3.296 \AA$ for pristine phosphorene, $a_{1}=4.274 \AA, a_{2}=2.467 \AA$ for pristine graphene and $a_{1}=6.702 \AA, a_{2}=3.867 \AA$ for pristine silicene. The in-plane strains are measured as the change of $a_{1}$ and $a_{2}$ upon charge injection into phosphorene, graphene and silicene along the armchair and zigzag directions, respectively. The electromechanical responses of pristine phosphorene upon charge injection are depicted in Fig. 2, in which the electromechanical responses of graphene and silicene monolayer are also calculated for comparison. It is clear that phosphorene monolayer can generate ultrahigh expansion (36.6\%) along the armchair direction upon hole injection ( 0.13 e/atom), which is 14 times larger than that along the zigzag direction (2.6\%) (Fig. 2). The highly anisotropic electromechanical responses originate from the remarkable structural and mechanical anisotropies of

${ }^{1}$ Department of Engineering Mechanics, School of Civil Engineering, Wuhan University, Wuhan, Hubei 430072, China. ${ }^{2}$ State Key Laboratory of Superlattices and Microstructures, Institute of Semiconductors, Chinese Academy of Sciences, Beijing 100083, China. ${ }^{\circledR}$ email: enlaigao@whu.edu.cn; ze.liu@whu.edu.cn 
a

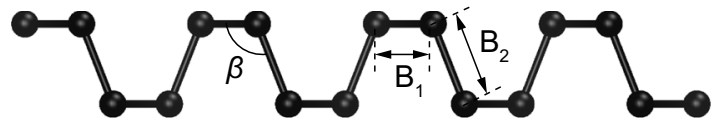

b

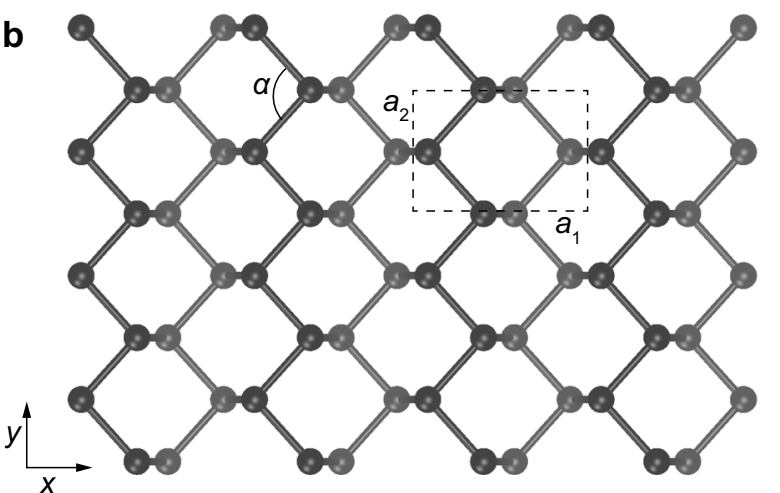

C

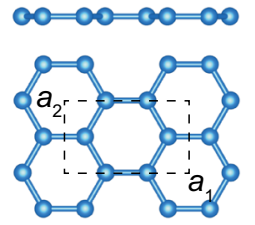

d

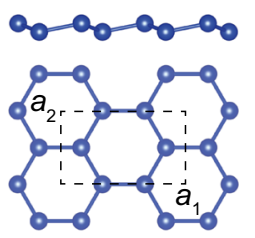

Fig. 1 Atomic configurations of phosphorene, graphene and silicene. a Side and $\mathbf{b}$ top views of monolayer phosphorene, $\mathbf{c}$ graphene and d silicene, respectively. The unit cells with lattice parameters of $a_{1}$ and $a_{2}$ are denoted by the dash lines. These labels of $B_{1-2}$ and $a-\beta$ in (a-b) denote two types of P-P bonds and two types of P-P-P angles in phosphorene, respectively.

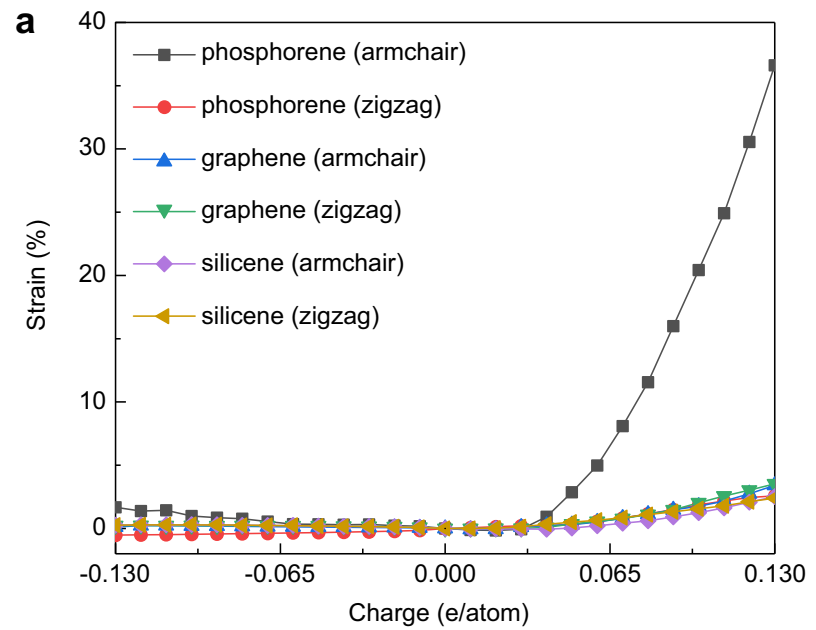

b

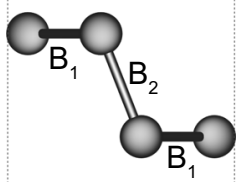

C

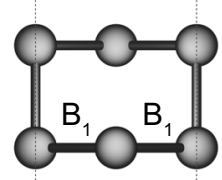

Fig. 2 Electromechanical strain responses of phosphorene, graphene and silicene. a Electromechanical strain responses of pristine phosphorene upon injecting electron (negative) and hole (positive) along the armchair and zigzag directions, respectively, compared with that of pristine graphene and silicene. (b) and (c) are the side views of phosphorene from the armchair and zigzag directions, respectively.

phosphorene ${ }^{23-25}$. Furthermore, the charge-induced maximum actuation strain of phosphorene is remarkably larger than that of graphene, silicene, and even the maximum value reported of GO $(28.2 \%)^{20,21}$, indicating that monolayer phosphorene exhibits an ultrahigh actuation performance. Upon low concentration hole injection (e.g., 0.02 e/atom), contraction (-0.16\%) along the armchair direction is initially observed followed by large expansions (up to 36.6\%) with the increasing of hole injection (Fig. 2). This phenomenon is also observed for the holeinduced $\mathrm{C}_{4} \mathrm{O}$-asym-unzip $\mathrm{GO}^{21}$, which is explained by considering the molecular orbitals associated with the $\mathrm{C}-\mathrm{O}-\mathrm{C}$ bonds. The hole-induced maximum strain of phosphorene is about 10-14 times greater in magnitude than that of monolayer graphene (3.5\%) and silicene $(2.5 \%)$ upon charging 0.13 e/atom in this work. Whereas, electron-induced contraction in the zigzag direction is distinctly different from the expansion of pristine graphene and silicene, and it is contrary to the quantummechanical actuation of covalent carbon materials, such as carbon nanotubes and graphene, where injected electron is believed to fill antibonding states and thus induces interatomic bond length expansions ${ }^{15,27,28}$.

Insights into the mechanism of electromechanical strain response To understand the mechanism of unusual electromechanical strain response in phosphorene, we calculated the interatomic deformations upon charge injection projected along the armchair and zigzag directions, respectively (Fig. $2 \mathrm{~b}-\mathrm{c}$ ). As shown in Table 1, upon 0.13 e/atom hole injection, phosphorene undergoes a net interatomic expansion with bond $B_{2}$ expanding by a considerable amount $(0.235 \AA)$ due to the increment of bond angle $\beta$ of $12.41^{\circ}$. $B_{1}$ shows neglectable contraction $(-0.009 \AA)$ due to the decreasing $\left(3.87^{\circ}\right)$ of the bond angle $a$, yet this is more offset by the large expansion of bond $B_{2}$. On the other hand, it is clear that $B_{2}$ shows a large expansion $(0.906 \AA)$ along the armchair direction but zero expansion along the zigzag direction. In spite of the projected deformation of $B_{1}(-0.062 \AA)$ along the armchair direction, the overall expansion of the unit cell along the armchair is $0.782 \AA$, leading to the massive expansion of $36.6 \%$. While the projected 
Table 1. The interatomic and its projected deformations $(\AA)$ along the armchair and zigzag directions of phosphorene upon change injection.

\begin{tabular}{llcrr}
\hline Charge Injection & & Interatomic & Armchair & Zigzag \\
\hline Hole (0.13 e/atom) & $\mathrm{B}_{1}$ & -0.009 & -0.062 & 0.042 \\
& $\mathrm{~B}_{2}$ & 0.235 & 0.906 & 0.000 \\
& total & 0.217 & 0.782 & 0.084 \\
Electron (-0.13 e/atom) & $\mathrm{B}_{1}$ & -0.005 & 0.001 & -0.009 \\
& $\mathrm{~B}_{2}$ & 0.034 & 0.035 & 0.000 \\
& total & 0.024 & 0.037 & -0.018 \\
\hline
\end{tabular}

deformation of $B_{1}$ along the zigzag direction is only $0.042 \AA$, resulting in the total expansion of the unit cell of $0.084 \AA(2.6 \%)$ along the zigzag direction. Based on these observations (Fig. 2), we further increased the injected electron (up to -0.13 e/atom), expansion of $1.7 \%$ and contraction of $-0.6 \%$ along the armchair and zigzag directions, respectively, were observed. Similarly, the interatomic projected deformations along the armchair and zigzag directions upon -0.13 e/atom electron injection were determined (Table 1). Such small increment of bond angle $\beta$ of $0.445^{\circ}$ leads to a small expansion of $B_{2}$ with a value of $0.034 \AA$, whereas the small reduction $\left(-0.357^{\circ}\right)$ of bond angle a causes a small contraction $(-0.005 \AA)$ of $B_{1}$.

To provide further insights into the essential physics of the electromechanical strain response of phosphorene upon charge injection, the excess charge density distribution upon charge injection (e.g., $\pm 0.02, \pm 0.06$, and \pm 0.13 e/atom) was calculated. As shown in Fig. 3a-b and Supplementary Fig. 2, we found that there is excess charge distributed in phosphorene, suggesting that the charge density of phosphorene has been redistributed. To show the influence of the excess charge on the bonds and angles of phosphorene, the evolutions of bonds $\left(B_{1}, B_{2}\right)$ and angles $(a, \beta)$ were plotted in Fig. 3c. Upon 0.13 e/atom injection (Fig. 3b and Supplementary Fig. 2d), the excess hole (shown in green) aggregates around $\mathrm{P}$ atoms and in $\mathrm{B}_{2}$, resulting in a remarkable repulsive interaction (red arrows) as illustrated in Fig. 3d. Hence, the $\beta$ and $B_{2}$ exhibit significant deformation. Besides, we found that there is some excess electron (shown in yellow) assigned upon $\mathrm{P}$ atoms and in $\mathrm{B}_{1}$, which results in a slight contraction (blue arrows) of $B_{1}$ (Fig. $3 d$ ). Upon electron injection of -0.13 e/atom (Fig. 3a and Supplementary Fig. 2a), the excess charge density shows about half of that upon hole injection of 0.13 e/atom, but it exhibits very smaller deformations of $B_{2}$ and $\beta$ than those upon hole injection of 0.13 e/atom. In addition to the interaction between excess charge, mechanical tests of the charged phosphorene demonstrate that the in-plane stiffness of hole charged phosphorene significantly decreases with increasing of hole injection, but the in-plane stiffness of electron charged phosphorene almost keeps constant with increasing of electron injection (Supplementary Fig. 3), suggesting that hole injection (depletion of electron) significantly weakens the structure of phosphorene. Therefore, the repulsive interaction of excess charge and the softening of phosphorene upon hole injection combinedly help us to understand the large actuation strain response in phosphorene.

Furthermore, we probe the influence of charge injection on the electronic structure of phosphorene. The projected densities of states (PDOS) upon hole injection and the corresponding excess charge density distributions were plotted in Fig. 4 and Supplementary Fig. 2. It can be found that the P atom has zero $s$ and $p$-DOS close to the Fermi level $(0 \mathrm{eV})$ before charge injection. However, for low concentration hole injection (0.02 e/atom), it's clear to find that $\mathrm{P}$ atom has non-zero $s$ - and $p$-DOS near the
Fermi level, which is further verified by the excess charge density distributions (Fig. 4c and Supplementary Fig. 2e and 2f), indicating the shift of Fermi level with the value of $-4.7 \mathrm{eV}$. As further injecting hole (0.06 e/atom), the calculated shift value can be $-13.6 \mathrm{eV}$. Conversely, as electron injection, the Fermi level would shift to left, e.g., -0.02 e/atom, the shift value is calculated as $3.3 \mathrm{eV}$. Furthermore, the band structures of phosphorene with electron and hole injection were calculated as depicted in Supplementary Fig. 4. Clearly, without charge injection, pristine phosphorene shows a direct band-gap of $0.84 \mathrm{eV}$, which is consistent with the previous report $(0.89 \mathrm{eV})$ at the PBE level ${ }^{24}$. As upon electron injection, we find that the Fermi energy level shifts to the conduction band corresponding to the right shift of that in PDOS. Electron injection induces the decreasing of the band-gap, e.g. phosphorene shows a band-gap of $0.66 \mathrm{eV}$ under $-0.06 \mathrm{e} /$ atom injection (Supplementary Fig. 4b). While hole injection induces the Fermi energy shift to valence band as well as the enlarging of the band-gap of $1.06 \mathrm{eV}$ upon 0.06 e/atom injection (Supplementary Fig. 4e). Additionally, based on Fermi-level shift value measured from the integrated density of states as used in previous work ${ }^{20,29}$, the voltages required to inject charge $( \pm 0.13$ e/atom) into phosphorene are estimated as about 1.1-3.0 V that is much lower than the withstand voltage of phosphorene ${ }^{30}$, suggesting that the maximum charge explored in this work is within the scope of security permission.

Structural integrity of phosphorene under the combined electromechanical loading

The mechanical stability of phosphorene under the combined electromechanical loading plays an essential role for practical applications. Herein, uniaxial tensile tests of the pristine and charged phosphorene were implemented (Fig. 5). It can be found that the fracture strain $\left(\varepsilon_{\max }\right.$, defined as the change of length divided by the actuated length of charged phosphorene) of electron charged phosphorene is larger than that of pristine phosphorene, while $\varepsilon_{\max }$ of hole charged phosphorene is smaller than that of pristine phosphorene (Supplementary Table 1). The increased fracture strain of phosphorene upon electron injection can be attributed to the excessive electron and the tiny actuation strain that almost has no effect on the capacity of structural deformation, while the reduced fracture strain of phosphorene upon hole injection can be attributed to the depletion of electron and the large actuation strain that exhausts the capacity of structural deformation (Fig. 3c). Herein, it should be noted that even upon hole injection of 0.13 e/atom, phosphorene still possesses a fracture strain of $4.4 \%$, indicating that the structural integrity is maintained under external mechanical loading.

\section{Actuation stress and volumetric work density of phosphorene}

To assess the feasibility of phosphorene as the building block for constructing actuators, the actuation stress and volumetric work density $\left(W_{\text {vol }}\right)$ of phosphorene as a function of charge injection are investigated. To be specific, the actuation stresses are calculated for the configurations with charge injection but fixed lattice constants of charge-free equilibrium configuration (Supplementary Fig. 5a), which generates high actuation stresses of 7.0 and $4.1 \mathrm{GPa}$ along the armchair and zigzag directions, respectively. Herein, the thickness of $5.55 \AA$ of phosphorene was adopted ${ }^{23}$. The actuation stress of charged phosphorene is lower than the reported GO $(>100 \mathrm{GPa})^{20}$ due to the smaller stiffness $(21.7 \mathrm{~N} / \mathrm{m}$ in the armchair direction and $89.9 \mathrm{~N} / \mathrm{m}$ in the zigzag direction) of phosphorene than that of GO $(\sim 204 \mathrm{~N} / \mathrm{m}$ in the armchair direction and $\sim 321 \mathrm{~N} / \mathrm{m}$ in the zigzag direction) ${ }^{31}$. Based on the method used in previous work $^{32,33}, W_{\mathrm{vol}}$ of phosphorene was calculated (Supplementary Fig. $5 b)$. Upon charge injection of -0.13 and 0.13 e/atom, $W_{\text {vol }}$ were calculated as 0.43 and $207.7 \mathrm{~J} / \mathrm{cm}^{3}$ along the zigzag and armchair directions, respectively, corresponding to the actuation strains of 
a<smiles>CO[AsH2]OC</smiles>

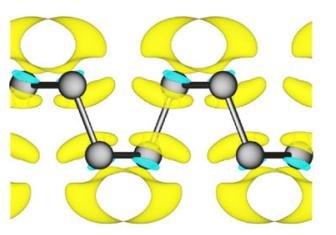

C

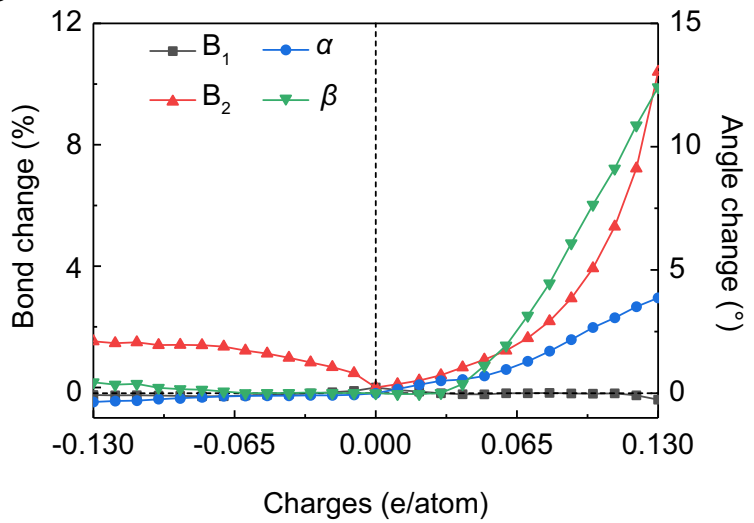

b

0.13 e/atom

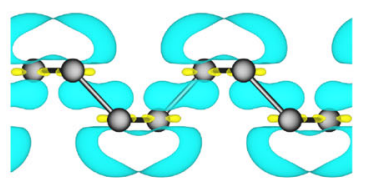

5

3
$\frac{1}{0}$
$\frac{0}{1}$
0
0
0
0
0
0
0 d
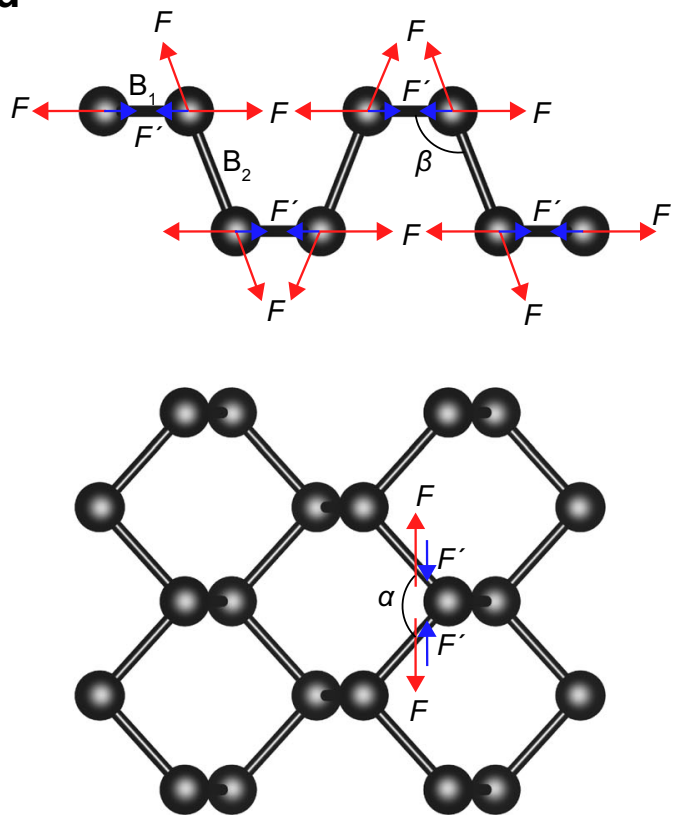

Fig. 3 Excess charge density distribution and interatomic responses of phosphorene. The side views of the excess charge density distribution of phosphorene upon charge injection of (a) -0.13 e/atom and (b) 0.13 e/atom at the iso-surface values of 0.0003 and 0.0006 e/ Bohr $^{3}$, respectively. Color coding of yellow and green represents excess electron and hole, respectively. $\mathbf{c}$ The evolutions of the bonds ( $B_{1}$ and $B_{2}$ ) and the angles ( $a$ and $\beta$ ) as a function of injected charge. $\mathbf{d}$ Illustration of the repulsion force ( $F$, the red arrows) and attraction force ( $F^{\prime}$, the blue arrows) from the Coulombic interactions generated by the excess electron-electron (hole-hole) and electron-hole, respectively.

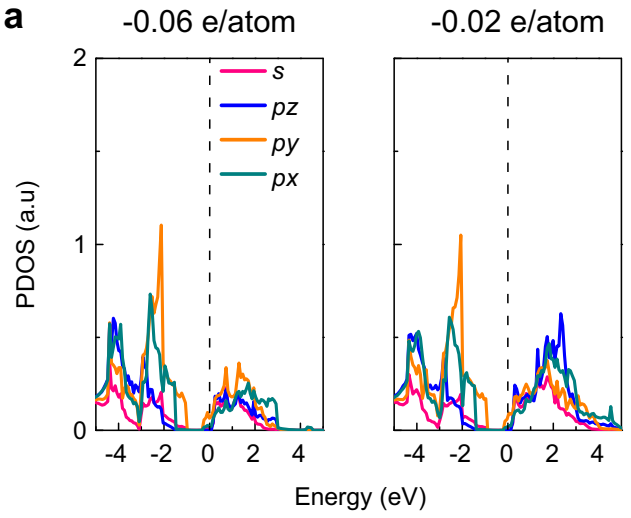

b -0.06 e/atom

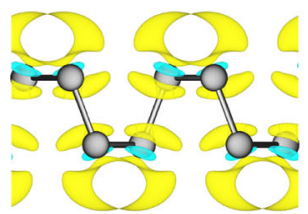

-0.02 e/atom

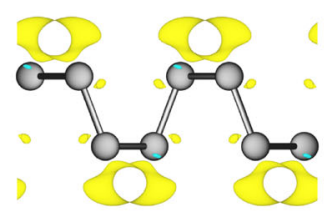

\subsection{0 e/atom}

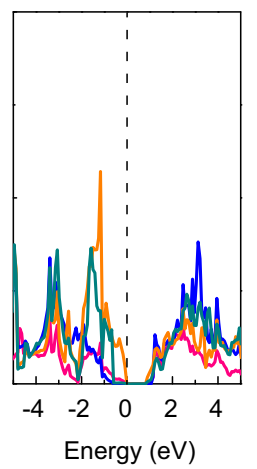

0.02 e/atom

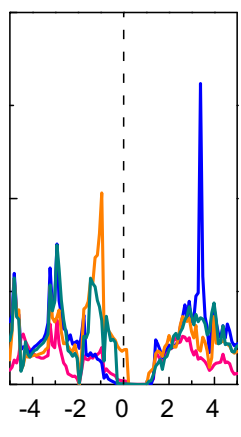

0.06 e/atom

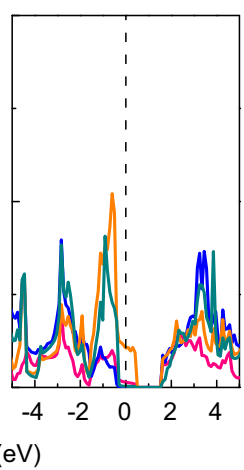

C

0.02 e/atom

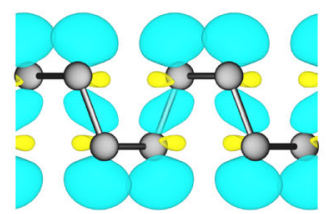

0.06 e/atom

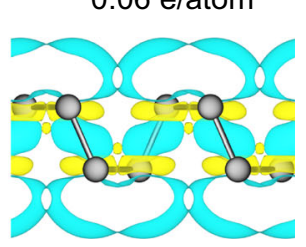

Fig. 4 Electronic properties of charged phosphorene. a PDOS of phosphorene upon charge injection of $-0.06,-0.02,0,0.02$, and 0.06 e/ atom, respectively. The calculated excess charge density distribution of phosphorene upon (b) electron and (c) hole injection at an iso-surface of $0.0002 \mathrm{e} / \mathrm{Bohr}^{3}$. Color coding of yellow and green represents excess electron and hole, respectively.

$1.67 \%$ and $36.6 \%$ along the armchair direction. The maximum $W_{\text {vol }}$ of phosphorene upon hole injection is larger than that of graphene $\left(35.3 \mathrm{~J} / \mathrm{cm}^{3}\right.$ ) and silicene $\left(3.16 \mathrm{~J} / \mathrm{cm}^{3}\right.$ ) (Supplementary Fig. $5 \mathrm{~b}$ ), mainly resulting from its large actuation strain. Compared to other well studied actuation materials (Supplementary Table 2), the maximum $W_{\mathrm{vol}}$ of phosphorene is approximately 1.4 times greater than that of GO $\left(144.1 \mathrm{~J} / \mathrm{cm}^{3}\right)^{21}$, and about 6-700 times greater than the CNT-based actuation material ${ }^{32}$ and the widely used ferroelectric materials ${ }^{33}$. The gravimetric work density $\left(W_{\mathrm{g}}\right)$ can be calculated as $W_{\mathrm{vol}} / \rho$, where $\rho$ is the density. The calculation demonstrated that phosphorene possesses an ultrahigh $W_{\mathrm{g}}$ of $\sim 1076.2 \mathrm{~J} / \mathrm{g}$ upon 0.13 e/atom injection, which is much larger than 

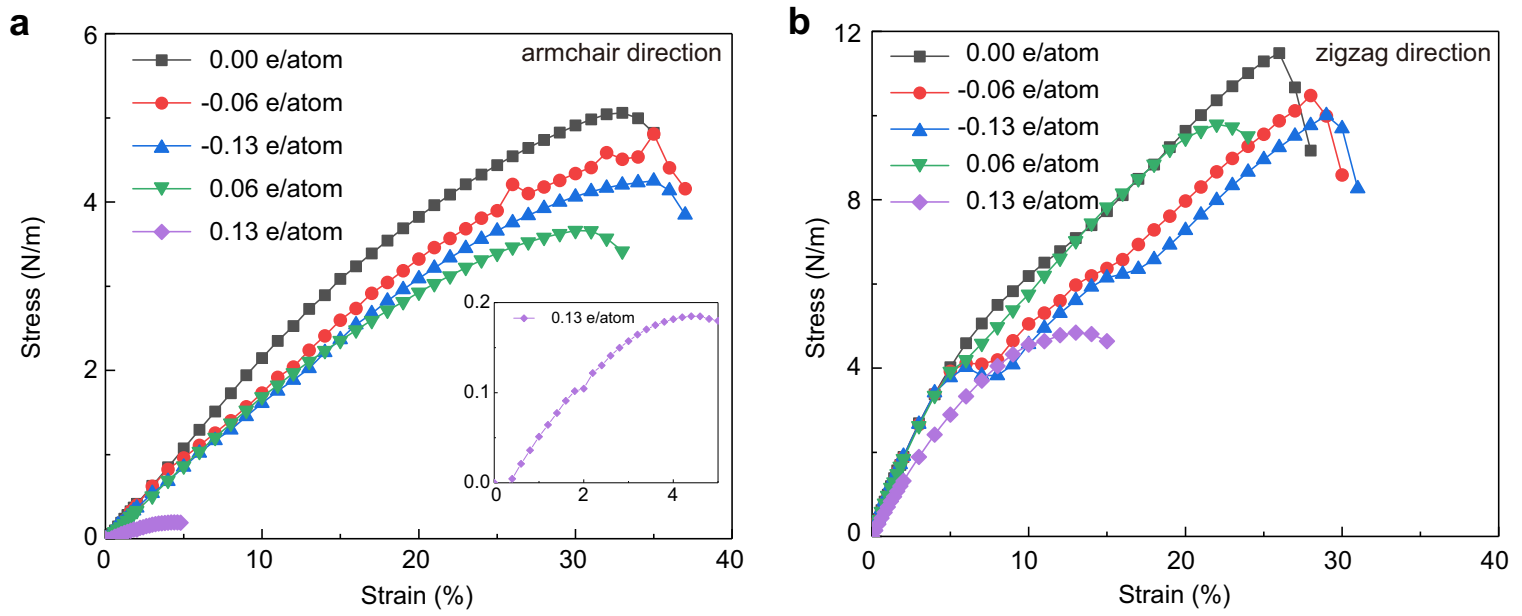

Fig. 5 Electromechanical stress response of phosphorene. Stress-strain curves of charged and pristine phosphorene along (a) the armchair and (b) zigzag directions, respectively. Inset of (a) is an enlarged view for the case upon hole injection of 0.13 e/atom.

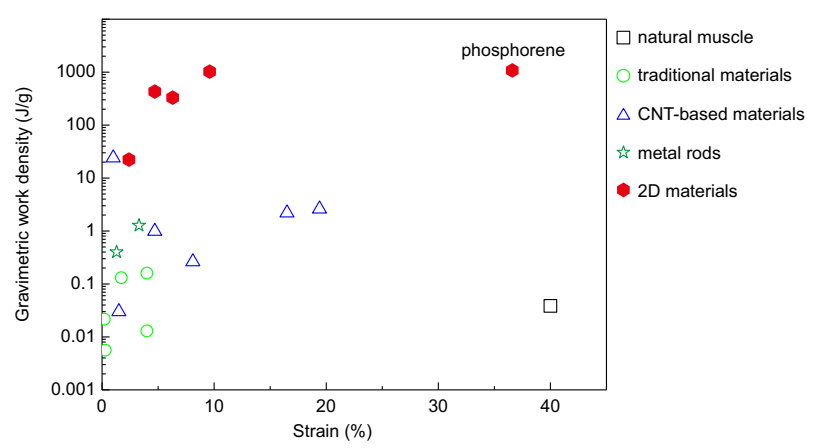

Fig. 6 Gravimetric work density and maximum actuation strain of phosphorene. The gravimetric work density and maximum actuation strain of phosphorene, comparing with other well-known actuation materials. All data are summarized in Supplementary Table 2.

natural muscle, CNT-based materials and other traditional materials (Supplementary Table 2 and Fig. 6). Considering the extreme large actuation strain, remarkable actuation stress and ultrahigh work density, phosphorene holds great promise in the applications of electromechanical actuators. Herein, it should be noted that $W_{\text {vol }}$ and $W_{\mathrm{g}}$ were calculated using the in-plane stiffness (Young's modulus) deduced from the pristine materials (Supplementary Note 2). However, our above calculations demonstrate that, the inplane stiffness of the charged phosphorene shows a significant reduction (Supplementary Fig. 3) upon hole injection. Therefore, $W_{\text {vol }}$ and $W_{\mathrm{g}}$ can be seen as nominal values. The realistic actuation stress $\sigma_{\mathrm{r}}$ and $W_{\text {vol-r }}$ are obtained as shown in Supplementary Fig. 6 (see Supplementary Note 2 for details). The maximum $W_{\text {vol-r }}$ $\left(154.1 \mathrm{~J} / \mathrm{cm}^{3}\right)$ and $W_{\mathrm{g}-\mathrm{r}}(798.4 \mathrm{~J} / \mathrm{g})$ are slightly smaller than their nominal values $\left(207.7 \mathrm{~J} / \mathrm{cm}^{3}\right.$ and $1076.2 \mathrm{~J} / \mathrm{g}$, respectively), resulting from the reduced in-plane stiffness.

Additionally, it is worth mentioning that we investigated the electromechanical response of free-standing monolayer phosphorene in this work, which can be seen as the ideal electromechanical performance of phosphorene. In practical applications, the pristine phosphorene is unstable upon exposure to air, and thus phosphorene-based devices should be protected by other materials to avoid degradation. However, the large actuation strain of phosphorene upon charge injection would induce significant mismatch with other materials. Fortunately, robust superlubricity of layered heterojunctions was recently reported ${ }^{34}$. Hence, future efforts should be directed, but not limited at discovering atom-smooth substrates having incommensurate contacting surface and thus ultralow sliding friction with phosphorene, which may make phosphorene almost freely actuate upon charge injection. In this way, the effect of substrate on the strain output of phosphorene would be reduced.

\section{DISCUSSION}

In this work, the electromechanical performance of pristine phosphorene was systematically investigated by using firstprinciples calculations. It was demonstrated that phosphorene possesses excellent electromechanical actuation performance. The maximum actuation strain, stress, and volumetric work density of phosphorene are as high as $36.6 \%, 7.0 \mathrm{GPa}$, and $207.7 \mathrm{~J} / \mathrm{cm}^{3}$, respectively. The underlying mechanism was then explored and well explained by the analysis of the atomic structure, electronic structure and mechanics. Moreover, we found that the charge injection has a significant impact on the electronic properties of monolayer phosphorene: hole injection enlarges the band-gap while electron injection narrows the band-gap. Finally, uniaxial tensile tests of charged phosphorene demonstrate the remarkable mechanical stability of phosphorene under the combined electromechanical loading. Our findings show that phosphorene holds a great promise for constructing nanoelectromechanical actuators.

\section{METHODS}

We explored the electromechanical performance of phosphorene, graphene, and silicene by performing first-principles calculations in the framework of plane-wave basis set based density functional theory (DFT) methods. The Perdew-Burke-Ernzerhof (PBE) parameterization ${ }^{35}$ of the generalized gradient approximation (GGA) ${ }^{36}$ was used for the exchangecorrelation functional. To correct the dispersion interactions, additional van der Walls correction by using DFT-D3 method was considered ${ }^{37}$. Projector augmented wave potentials were used to treat ion-electron interactions ${ }^{38}$. All calculations were carried out by the Vienna Ab initio Simulation Package (VASP) ${ }^{39,40}$. For all results presented in this work, energy cut-off of $130 \%$ of the default value was used to ensure the accuracy. MonkhorstPack grid $k$-points ${ }^{41}$ with the density $>40 \AA$ were used for the Brillouin zone sampling in all calculations. With the conjugated gradient algorithm, all structures were fully relaxed to their ground states before charge injection. For the geometry relaxation, the convergence criteria is less than $0.01 \mathrm{eV} / \AA \AA$ for the force on each atom. Previous calculation of graphene and GO suggests a vacuum layer of $60 \AA$ is necessary for the calculations of electromechanical behaviors to minimize the interlayer electrostatic 
interactions ${ }^{19,21}$. Considering a balance between computational accuracy and efficiency (Supplementary Note 1 and Supplementary Fig. 1), a thick vacuum layer of $70 \AA$ was adopted and held constant through modifying the VASP code to relax the cell within the basal plane of phosphorene only to minimize the interaction between periodic images. Additionally, considering that the homogeneous background charge also contributes to the total energy of the system, we adopted the energy correction method proposed by Reed et al. ${ }^{42}$ to calculate the total energy of the charged phosphorene (Supplementary Fig. 7).

\section{DATA AVAILABILITY}

The data that support the findings of this study are available from the corresponding author upon reasonable request.

\section{CODE AVAILABILITY}

All codes of this current study are available from the corresponding author upon reasonable request.

Received: 24 September 2019; Accepted: 3 March 2020; Published online: 20 March 2020

\section{REFERENCES}

1. Mirvakili, S. M. \& Hunter, I. W. Artificial muscles: mechanisms, applications, and challenges. Adv. Mater. 30, 1704407 (2018).

2. Mirvakili, S. M. et al. Niobium nanowire yarns and their application as artificial muscles. Adv. Funct. Mater. 23, 4311-4316 (2013).

3. Duduta, M. et al. Realizing the potential of dielectric elastomer artificial muscles. Proc. Natl Acad. Sci. USA 116, 2476-2481 (2019).

4. Mirvakili, S. M. \& Hunter, I. W. Multidirectional artificial muscles from nylon. Adv. Mater. 29, 1604734 (2017).

5. Mu, J. et al. Sheath-run artificial muscles. Science 365, 150-155 (2019).

6. Close, R. I. Dynamic properties of mammalian skeletal muscles. Physiol. Rev. 52, 129-197 (1972).

7. Close, R. \& Hoh, J. F. Y. Influence of temperature on isometric contractions of rat skeletal muscles. Nature 217, 1179-1180 (1968).

8. Lv, S. et al. Designed biomaterials to mimic the mechanical properties of muscles. Nature 465, 69-73 (2010).

9. Winzeler-Merçay, U. \& Mudie, H. The nature of the effects of stroke on trunk flexor and extensor muscles during work and at rest. Disabil. Rehabil. 24, 875-886 (2002).

10. Madden, J. D. W. et al. Artificial muscle technology: physical principles and naval prospects. IEEE J. Ocean. Eng. 29, 706-728 (2004).

11. Hollerbach, J. M. H. I. W. \& Ballantyne, J. A comparative analysis of actuator technologies for robotics. Robot. Rev. 2, 299-342 (1992).

12. Jiang, T. Y., Ng, T. Y. \& Lam, K. Y. Optimization of a piezoelectric ceramic actuator. Sens. Actuators A: Phys. 84, 81-94 (2000).

13. Haines, C. S. et al. Artificial muscles from fishing line and sewing thread. Science 343, 868-872 (2014).

14. Novoselov, K. S. et al. Electric field effect in atomically thin carbon films. Science 306, 666-669 (2004).

15. Sun, G., Kürti, J., Kertesz, M. \& Baughman, R. H. Dimensional changes as a function of charge injection in single-walled carbon nanotubes. J. Am. Chem. Soc. 124, 15076-15080 (2002).

16. Gao, E. \& Xu, Z. Thin-Shell thickness of two-dimensional materials. J. Appl. Mech. 82, 121012 (2015).

17. Gao, E. et al. Mechanical exfoliation of two-dimensional materials. J. Mech. Phys. Solids 115, 248-262 (2018).

18. Jia, X., Liu, Z. \& Gao, E. Bio-inspired self-folding strategy to break the trade-off between strength and ductility in carbon-nanoarchitected materials. npj Comput. Mater. 6, 13 (2020).

19. Rogers, G. W. \& Liu, J. Z. Graphene actuators: quantum-mechanical and electrostatic double-layer effects. J. Am. Chem. Soc. 133, 10858-10863 (2011).

20. Rogers, G. W. \& Liu, J. Z. High-performance graphene oxide electromechanical actuators. J. Am. Chem. Soc. 134, 1250-1255 (2012).

21. Rogers, G. W. \& Liu, J. Z. Monolayer graphene oxide as a building block for artificial muscles. Appl. Phys. Lett. 102, 021903 (2013).

22. Liu, H. et al. Phosphorene: an unexplored 2D semiconductor with a high hole mobility. ACS Nano 8, 4033-4041 (2014).

23. Wei, Q. \& Peng, X. Superior mechanical flexibility of phosphorene and few-layer black phosphorus. Appl. Phys. Lett. 104, 251915 (2014).
24. Wang, L., Kutana, A., Zou, X. \& Yakobson, B. I. Electro-mechanical anisotropy of phosphorene. Nanoscale 7, 9746-9751 (2015).

25. Li, R., Shao, Q., Gao, E. \& Liu, Z. Elastic anisotropy measure for two-dimensional crystals. Extreme Mechanics Lett. 100615 (2019).

26. Liu, F., Ming, P. \& Li, J. Ab initio calculation of ideal strength and phonon instability of graphene under tension. Phys. Rev. B 76, 064120 (2007).

27. Verissimo-Alves, M., Koiller, B., Chacham, H. \& Capaz, R. B. Electromechanical effects in carbon nanotubes: $A b$ initio and analytical tight-binding calculations. Phys. Rev. B 67, 161401 (2003).

28. Sun, G., Kertesz, M., Kürti, J. \& Baughman, R. H. Dimensional change as a function of charge injection in graphite intercalation compounds: a density functional theory study. Phys. Rev. B 68, 125411 (2003).

29. Fang, T., Konar, A., Xing, H. \& Jena, D. Carrier statistics and quantum capacitance of graphene sheets and ribbons. Appl. Phys. Lett. 91, 092109 (2007).

30. Yu, X., Zhang, S., Zeng, H. \& Wang, Q. J. Lateral black phosphorene P-N junctions formed via chemical doping for high performance near-infrared photodetector. Nano Energy 25, 34-41 (2016).

31. Xu, Z. \& Xue, K. Engineering graphene by oxidation: a first-principles study. Nanotechnology 21, 045704 (2009).

32. Baughman, R. H. Carbon nanotube actuators. Science 284, 1340-1344 (1999).

33. Zhang, Q. M., Bharti, V. \& Zhao, X. Giant electrostriction and relaxor ferroelectric behavior in electron-irradiated poly(vinylidene fluoride-trifluoroethylene) copolymer. Science 280, 2101-2104 (1998).

34. Song, Y. et al. Robust microscale superlubricity in graphite/hexagonal boron nitride layered heterojunctions. Nat. Mater. 17, 894-899 (2018).

35. Perdew, J. P., Burke, K. \& Ernzerhof, M. Generalized gradient approximation made simple. Phys. Rev. Lett. 77, 3865-3868 (1996).

36. Filippi, C., Singh, D. J. \& Umrigar, C. All-electron local-density and generalizedgradient calculations of the structural properties of semiconductors. Phys. Rev. B 50, 14947 (1994).

37. Grimme, S., Antony, J., Ehrlich, S. \& Krieg, H. A consistent and accurate ab initio parametrization of density functional dispersion correction (DFT-D) for the 94 elements H-Pu. J. Chem. Phys. 132, 154104 (2010).

38. Blöchl, P. E. Projector augmented-wave method. Phys. Rev. B 50, 17953 (1994).

39. Kresse, G. \& Furthmüller, J. Efficient iterative schemes for ab initio totalenergy calculations using a plane-wave basis set. Phys. Rev. B 54, 11169 (1996).

40. Kresse, G. \& Furthmüller, J. Efficiency of ab-initio total energy calculations for metals and semiconductors using a plane-wave basis set. Comput. Mater. Sci. 6, 15-50 (1996).

41. Monkhorst, H. J. \& Pack, J. D. Special points for Brillouin-zone integrations. Phys. Rev. B 13, 5188 (1976).

42. Li, Y., Duerloo, K.-A. N., Wauson, K. \& Reed, E. J. Structural semiconductor-tosemimetal phase transition in two-dimensional materials induced by electrostatic gating. Nat. Commun. 7, 10671 (2016).

\section{ACKNOWLEDGEMENTS}

This work was supported by the National Natural Science Foundation of China (11872284, 11632009, and 11602175), and the Fundamental Research Funds for the Central Universities (413000091). The numerical calculations in this work have been done on the supercomputing system in the Supercomputing Center of Wuhan University.

\section{AUTHOR CONTRIBUTIONS}

E.G. conceived the idea. Z.L. provided advices on this work. B.W. and X.J. conducted the simulation and analysis. All authors participated in the interpretation of the data and the writing of the manuscript.

\section{COMPETING INTERESTS}

The authors declare no competing interests.

\section{ADDITIONAL INFORMATION}

Supplementary information is available for this paper at https://doi.org/10.1038/ s41524-020-0297-6.

Correspondence and requests for materials should be addressed to E.G. or Z.L.

Reprints and permission information is available at http://www.nature.com/ reprints 
Publisher's note Springer Nature remains neutral with regard to jurisdictional claims in published maps and institutional affiliations.

(c) Open Access This article is licensed under a Creative Commons Attribution 4.0 International License, which permits use, sharing, adaptation, distribution and reproduction in any medium or format, as long as you give appropriate credit to the original author(s) and the source, provide a link to the Creative
Commons license, and indicate if changes were made. The images or other third party material in this article are included in the article's Creative Commons license, unless indicated otherwise in a credit line to the material. If material is not included in the article's Creative Commons license and your intended use is not permitted by statutory regulation or exceeds the permitted use, you will need to obtain permission directly from the copyright holder. To view a copy of this license, visit http://creativecommons. org/licenses/by/4.0/.

c) The Author(s) 2020 